\title{
Correction: Problematic Internet Usage and Immune Function
}

Phil Reed, Rebecca Vile, Lisa A. Osborne, Michela Romano, Roberto Truzoli

There are errors in the author affiliations. The affiliations should appear as shown here: Phil Reed ${ }^{1}$, Rebecca Vile ${ }^{1}$, Lisa A. Osborne ${ }^{2}$, Michela Romano ${ }^{3}$, Roberto Truzoli ${ }^{3}$

1 Swansea University, Swansea, United Kingdom, 2 Abertawe Bro Morgannwg University Health Board, Swansea, United Kingdom, 3 Università degli Studi di Milano, Milan, Italy.

There is an error in Table 1. The second occurrence of the word "Gambling" in the first column should read "Gaming." Please see the corrected Table 1 here.

\section{G openaccess}

Citation: Reed P, Vile R, Osborne LA, Romano M, Truzoli R (2015) Correction: Problematic Internet Usage and Immune Function. PLoS ONE 10(10): e0140692. doi:10.1371/journal.pone.0140692

Published: October 9, 2015

Copyright: @ 2015 Reed et al. This is an open access article distributed under the terms of the Creative Commons Attribution License, which permits unrestricted use, distribution, and reproduction in any medium, provided the original author and source are credited. 
Table 1. Percentage of sample visiting websites of various forms, along with percentage male and females, and younger and older, participants visiting sites along with Phi coefficients.

\begin{tabular}{|c|c|c|c|c|c|c|c|}
\hline & Sample & Female & Male & Phi & $<30$ years & $30+$ years & Phi \\
\hline Social Networking & 95.0 & 97.0 & 92.9 & $.094^{*}$ & 96.6 & 91.6 & $.105^{*}$ \\
\hline Shopping/Banking & 87.1 & 90.6 & 83.2 & $.108^{* *}$ & 84.6 & 92.9 & $.115^{* *}$ \\
\hline Research & 82.2 & 83.0 & 81.1 & .023 & 84.6 & 76.8 & $.094^{*}$ \\
\hline Gambling & 70.3 & 70.2 & 70.8 & .007 & 70.8 & 69.9 & .012 \\
\hline TV and film & 67.3 & 64.2 & 70.8 & .071 & 68.0 & 65.8 & .022 \\
\hline Dating \& sexual & 65.0 & 59.2 & 73.3 & $.148 * * *$ & 66.0 & 65.8 & .002 \\
\hline News & 57.4 & 58.5 & 56.2 & .023 & 52.9 & 67.7 & $.139 * * *$ \\
\hline Content sharing & 46.5 & 44.5 & 48.8 & .042 & 46.0 & 47.7 & .016 \\
\hline Gaming & 28.3 & 20.0 & 37.5 & $.194 * * *$ & 27.4 & 30.3 & .030 \\
\hline Blogging & 16.8 & 15.5 & 18.3 & .038 & 14.3 & 22.6 & $.102^{*}$ \\
\hline Chat rooms & 9.8 & 1.9 & 16.7 & $.259 * * *$ & 6.3 & 14.8 & $.138 * * *$ \\
\hline
\end{tabular}

${ }^{*} p<0.05$

$* * p<0.01$

$* * *<0.001$.

doi:10.1371/journal.pone.0140692.t001

\section{Reference}

1. Reed P, Vile R, Osborne LA, Romano M, Truzoli R (2015) Problematic Internet Usage and Immune Function. PLoS ONE 10(8): e0134538. doi:10.1371/journal.pone.0134538 PMID: 26244339 